\title{
Impact of County Sociodemographic Factors and State Policy on Geographic Access to Behavior Analysts Among Children with Autism Spectrum Disorder
}

\author{
Marissa E. Yingling ${ }^{1}$ (D) Matthew H. Ruther ${ }^{2} \cdot$ Erick M. Dubuque $^{3} \cdot$ Bethany A. Bell $^{4}$ \\ Accepted: 8 February 2021 / Published online: 23 February 2021 \\ (c) The Author(s), under exclusive licence to Springer Science+Business Media, LLC part of Springer Nature 2021
}

\begin{abstract}
To examine the relationship between geographic access to Board Certified Behavior Analysts (BCBAs) among children with autism spectrum disorder (ASD) and county sociodemographic factors and state policy, we integrated publicly available data from the U.S. Department of Education's Civil Rights Data Collection, Behavior Analyst Certification Board's certificant registry, and U.S. Census. The study sample included U.S. counties and county equivalents (e.g., parishes, independent cities) in 49 states and D.C. $(\mathrm{N}=3040)$. Using GIS software, we assigned BCBAs to counties based on their residence, allocated children via school districts to counties, and generated per-capita children with ASD/BCBA ratios. We distributed counties into five categories based on these ratios: no BCBAs (reference), $\geq 31,21-30,11-20,>0-10$. We used a generalized logit model to conduct analyses. Highly affluent and urban counties had the highest access to BCBAs with odds ratio estimates for affluence ranging from 2.26 to 5.26. County-level poverty was positively associated with access, yet this relationship was moderated by urbanicity. Race-ethnicity and healthcare insurance coverage were negatively related to access. Other variables were not significant. Targeting non-urban and less affluent counties for provider recruitment and maintenance could most improve access to BCBAs. In addition to strategies specific to BCBAs for improving geographic access, traditional strategies used for other healthcare providers could be useful.
\end{abstract}

Keywords Board certified behavior analysts $\cdot$ Autism spectrum disorder $\cdot$ Geographic access $\cdot$ Behavior analysis

\section{Introduction}

Autism spectrum disorder (ASD) is a neurodevelopmental disorder characterized by impairments in social communication and social interaction and restricted and repetitive behaviors and interests (American Psychiatric Association 2013). The complex, heterogeneous symptoms of ASD

Marissa E. Yingling

marissa.yingling@louisville.edu

1 Kent School of Social Work, University of Louisville, 2217 S 3rd St, Oppenhimer Hall, Louisville, KY 40208, USA

2 College of Arts and Sciences, Department of Urban and Public Affairs, University of Louisville, Louisville, KY, USA

3 College of Education and Human Development, Department of Special Education, Early Childhood \& Prevention Science, University of Louisville, Louisville, KY, USA

4 College of Social Work, University of South Carolina, Columbia, SC, USA typically manifest before a child's third birthday (Baio et al. 2018) and require intensive, individualized treatments to ameliorate. Coverage for the diagnosis and treatment of ASD, including applied behavior analytic (ABA) services provided by Board Certified Behavior Analysts (BCBAs), is now mandated through state-regulated private and public healthcare insurance in all states (Centers for Medicare and Medicaid Services 2014; State Regulated Health Benefit Plans 2020). In their work with children with ASD, BCBAs may utilize three types of treatment models: consultation, focused, and early intensive behavioral intervention (comprehensive ABA). When the targeted behavior(s) are relatively minor in scope and can be addressed primarily by caregivers and teachers working as mediators under the direction of a BCBA, the consultation treatment model involves the smallest dosage of hours (1 to $10 \mathrm{~h}$ per week). The focused treatment model, which is utilized to treat more intensive behaviors that require assistance from the BCBA (e.g., self-injury, communication strategy) and often a team of behavior technicians whom the BCBA supervises, 
includes 10 to $25 \mathrm{~h}$ per week. A BCBA uses the comprehensive applied behavior analysis treatment model when attempting to change a child's developmental trajectory. This model is often highly intensive ( 25 to $40 \mathrm{~h}$ per week), targets all domains impacted by ASD (e.g., communication, social interactions, repetitive behaviors), and is delivered by behavior technicians overseen by a BCBA. The results of meta-analyses indicate that children who receive comprehensive ABA demonstrate significant improvements in cognitive, language, social communication, and daily living skills (Peters-Scheffer et al. 2011; Virués-Ortega 2010). In general, ABA services have demonstrated success in improving socially significant behavior with adolescents and young adults with ASD (National Autism Center 2015; Roth and DiGennaro-Reed 2014; US Department of Education, Institute for Education Sciences 2016; Wong et al. 2014).

Healthcare insurance coverage of ABA services is a necessary ingredient for ensuring that children with ASD have access to these services. Yet coverage is likely insufficient. In a recent systematic review on the U.S. healthcare workforce for children with ASD, investigators concluded that there is limited geographic availability of specialty providers and that there is a need for research that further explores the accessibility of this workforce (McBain et al. 2019). Largely in response to increased demand for ABA services for children with ASD (Carr \& Nosik 2017), the number of BCBAs in the U.S. has risen dramatically. In 1999, there were 28; by January 2019, twenty years later, the number of active BCBAs totaled 27,289 (Behavior Analyst Certification Board 2019a). This upward trend strongly suggests that the supply of providers for children with ASD is greater than ever. Approximately $68 \%$ of BCBAs work primarily with individuals with ASD, $12 \%$ work primarily in education (where children with ASD attend school), and $8 \%$ work primarily with individuals with developmental disabilities (which includes children with ASD) (Behavior Analyst Certification Board 2019a). Nevertheless, whether geographic access to BCBAs is equitable remains unclear.

Geographic access to healthcare providers is a long-standing challenge; populations who live in areas characterized by socioeconomic advantages typically fair better. Primary care providers tend to practice in more affluent areas and where there are already enough or too many providers (Davis et al. 2018; Shipman et al. 2011). Whereas $20 \%$ of the U.S. population resides outside of urban areas, only $12 \%$ of providers practice in rural areas, and the more rural a location, the fewer specialty providers (Rosenblatt et al. 2010). Indeed, in one southeastern state, more children with ASD who live in nonrural areas access recommended behavior support services than do children in rural areas, and children in non-rural areas travel fewer miles (14 miles) than rural families (36 miles) to receive services (Mello et al. 2016). The distributions of psychiatrists, psychologists, and psychiatric nurse practitioners also vary wildly by U.S. region (Andrilla et al. 2018). Furthermore, evidence suggests that sociodemographic factors are associated with access. For instance, race-ethnicity is associated with geographic access to primary care providers in both urban and rural areas (Brown et al. 2016; Ko et al. 2016) as well as to mental healthcare providers (Cummings et al. 2013).

Geographic access to healthcare is important for health outcomes. Greater supply of healthcare providers improves healthcare utilization (Guttmann et al. 2010) and patient outcomes, particularly in areas where there are very few providers (Goodman et al. 2002). Among children with ASD, those who live in an area with more neurologists or psychiatrists and children who live near a medical school are diagnosed earlier (Kalkbrenner et al. 2011). This literature suggests that investigating whether there are factors associated with geographic access to BCBAs is a critical first step to promoting optimal outcomes among children with ASD. To date, no research examines factors associated with geographic access to BCBAs in the U.S. Only one study examines the geographic distribution of BCBAs. Using descriptive statistics to explore Behavior Analyst Certification Board (BACB) certification trends between 1999 and 2014, researchers demonstrated that states with larger populations have a higher number of BACB certificants, and that compared to states of similar population size, three states - New Jersey, Massachusetts, and Connecticut - had higher per capita distribution of BACB certificants (Deochand and Fuqua 2016). Additional work is necessary to establish an understanding of the factors that are associated with geographic access to BCBAs.

The 2017 Interagency Autism Coordinating Committee strategic plan calls for research to improve and ensure equitable access, especially in high-poverty areas and traditionally underserved regions and populations. The committee also recognizes the pressing need to develop workforce capacity for ASD-related services. To this end, in the current study, we examine the relationship between geographic access to BCBAs among children with ASD and county-level sociodemographic factors and state healthcare insurance policy characteristics. We expect that counties with lower ASD/ BCBA ratios will: 1) be counties that are more socially and economically advantaged;2) be within states that passed a healthcare insurance mandate for coverage of ABA services earlier; and 3) be within states in which there is no age cap on ABA services in the state's healthcare insurance mandate.

\section{Method}

\section{Data}

The study sample included all U.S. counties and county equivalents (e.g., parishes, independent cities) in 49 states 
and D.C. $(\mathrm{N}=3040)$. We integrated publicly available data from the U.S. Department of Education's Civil Rights Data Collection (CRDC) and the BACB. The CRDC is biennial and tracks education and civil rights issues in public schools. We used the most recent CRDC data (2015-2016), which includes all local educational agencies in the U.S. (i.e., school districts, charter schools, juvenile justice facilities, alternative schools, and schools serving only students with disabilities) (United States Department of Education 2018). Children with educational disabilities are reported by their primary special education category. Thus, if a child has a primary diagnosis of ASD and a secondary diagnosis of intellectual disability (ID), the child is only included in the ASD count for that district. This study was exempt from the University Institutional Review Board, as the data were existing, public-use data.

The BACB registry includes BCBAs and Board Certified Behavior Analysts - Doctorate (BCBA-D). Both are equally qualified to provide services, so both were included in this analysis. The registry also includes Board Certified Assistant Behavior Analysts. Because a BCBA or BCBA-D must supervise these mid-level providers, we excluded them. The BACB registry includes all BCBAs with active and inactive certification (BCBAs renew certification every two years) and is updated monthly. We used the most recent month of BACB certificant data available at the time of the study and only included active certificants.

The CRDC and BACB data lack county designations. The CRDC provides a count of children with ASD by school district. We allocated children to counties based on the percentage of the district population that is within each county, using the Geographic Correspondence Engine provided by the Missouri Census Data Center. The BACB registry includes names, certification type, original date of certification, and city of residence. In typical cases where a city lies within one county, we assigned BCBAs to that county. In unusual cases where a city lies within multiple counties, we used the geographic information systems (GIS) method of areal interpolation (Goodchild and Lam 1980; Markoff and G 1973) to allocate BCBAs to counties based on the percentage of the city population that is within each county.

\section{Dependent Variable}

To measure geographic access, we completed two steps. First, for each county, we calculated the ASD/BCBA ratio as the quotient of the number of children with ASD divided by the number of BCBAs (i.e., lower numbers represent greater access). In the CRDC data, a school district with 2 or fewer children with ASD is represented as $\leq 2$. In these cases, we replaced $\leq 2$ with 1 , an impartial approach that is neither conservative (0) nor liberal (2). Next, we distributed counties into five categories based on these ratios: no
BCBAs (reference category), 31 or greater children with ASD per BCBA, 21 to 30 children with ASD per BCBA, 11 to 20 children with ASD per BCBA and greater than 0 to 10 children with ASD per BCBA. Initially, we used a Natural Breaks (Jenks) classification to symbolize ratios within counties. However, this method resulted in categories with too few counties. For instance, one category had only 11 counties. Therefore, we established categories that would better distribute counties while taking into consideration the BACB's caseload recommendation guidelines for children with ASD (Behavior Analyst Certification Board 2014). For instance, the BACB recommends that a BCBA with a focused treatment caseload serve between 10 to 15 clients when working alone and 16 to 24 clients when working with the support of one Board Certified Assistant Behavior Analyst (BCaBA). Yet this changes when treatment involves severe problem behavior or necessitates high supervisory oversight. The BACB also recommends that a BCBA with a comprehensive caseload oversee 6 to 12 clients or 12 to 16 clients when they have the support of one BCaBA. However, this recommendation changes based on case complexity and the skill and expertise of the behavior technicians who deliver most of the hours overseen by the BCBA (Behavior Analyst Certification Board 2014).

\section{Independent County Variables}

We obtained county-level sociodemographic and socioeconomic variables through the U.S. Census Bureau's American Community Survey program. Percent non-Hispanic white is the percent of white residents in the county (grand-mean centered). Poverty is a composite variable calculated as a mean $\mathrm{z}$-score computed from variables commonly used to measure poverty (Leventhal \& BrooksGunn 2003): percent of children below the federal poverty level, percent of households who received supplemental security income, cash public assistance income or supplemental nutrition assistance program benefits in the past 12 months, and percent of residents who are unemployed. Affluence, which is used to measure quality of a geographic area, is a z-score computed from variables often used to measure affluence (Leventhal and Brooks-Gunn 2003): median household income, percent of residents with professional/managerial employment, and percent of residents 25 years or older with a Bachelor's degree or higher. We measured affluence in addition to the more traditionally utilized measure of poverty given that the two variables measure distinct aspects of a geographic area and recent evidence that poverty and affluence differentially impact utilization of behavioral intervention among children with ASD (Yingling et al. 2019). No Healthcare Insurance is the percent of county residents under the age of 19 without healthcare insurance. Originally, we also 
included a variable measuring the percent of county residents under the age of 19 who were Medicaid eligible. However, this variable was strongly correlated with No Healthcare Insurance, so we removed it from analyses.

To measure urbanicity, we used the U.S. Department of Agriculture's Rural-Urban Continuum Codes. These codes categorize counties by "the population size of their metro area and nonmetropolitan counties by degree of urbanization and adjacency to a metro area" (United States Department of Agriculture 2019). We created dummy variables for counties within metropolitan statistical areas (MSAs), urban non-MSAs, and rural counties.

\section{Independent State Variables}

We included two state-level variables in our analyses. The continuous variable years since insurance mandate is expressed as the difference between the year data were collected (2019) and the year a state signed into law a healthcare insurance mandate to cover ASD diagnosis and treatment. For instance, if a state adopted a mandate in 2014, five years passed since the mandate became law (2019-2014 $=5$ years). This variable is included to account for the addition of a significant funding source for ABA services that BCBAs are credentialed to provide. The dichotomous variable no age cap expresses whether a state's mandate capped the age at which an individual with ASD could receive ABA services $(1=$ no age cap, $0=$ age cap). This variable is included given evidence that age caps reduce the probability of ASD-specific healthcare service utilization (Kennedy-Hendricks et al. 2018). In states without age caps there may be a higher number of potential clients for whom BCBAs can provide ABA services. To identify the year when a state implemented a mandate and whether a state mandate included an age cap for ABA services, we collected information from Autism Speaks and reviewed information from the original mandate legislation provided by the National Conference on Legislatures for year 2018 (National Conference of State Legislatures 2019).

\section{Missing Data}

Of the 3142 U.S. counties and county equivalents, 3040 had no missing data. We excluded Iowa's 99 counties because the state did not report data on children with ASD in their special education counts. Three additional counties-one in Virginia, one in Alaska, and one in South Dakota-were missing CRDC data. These counties were removed for a final sample of 3,040. Missing data across all counties and all variables was $0.004 \%$.

\section{Statistical Analyses}

We conducted analyses using SAS® 9.4. Initially, we used two-level hierarchical generalized linear models (i.e., counties nested in states). However, due to the low number of counties within some states and multiple categories of the dependent variable, the models did not converge. Therefore, we estimated one contextual generalized logit model that included all county- and state-level variables. To account for counties being nested in states our model was estimated using PROC SURVEY LOGISTIC using an alpha level of 0.05 . Furthermore, we analyzed the dependent variable as a multinomial variable and not a continuous variable for two reasons. First, we were interested in examining the impact of county- and state-level factors on the discrete comparisons between different categories of geographic access versus examining the impact of our independent variables over all levels of our outcome. Second, we aimed to maximize the usefulness of study findings for policy makers.

\section{Results}

Table 1 includes descriptive statistics for geographic access to BCBAs among children with ASD, county sociodemographics, and state healthcare insurance policy characteristics. More than half of all U.S. counties in the sample had no BCBAs $(n=1611)$, and $17.5 \%$ of counties were in the highest access ASD/BCBA ratio category $(>0$ to 10 ASD/BCBA; $\mathrm{n}=533$ ). Table 2 presents estimates for the generalized logit models. An increase in poverty was related to the odds of being in the two highest access ASD/BCBA ratio categories (OR 1.63, 95\% CI 1.19 to 2.23 and OR $1.76,95 \%$ CI 1.26 to 2.44 , respectively). A similar pattern was observed with affluence. Overall, as affluence increased the odds of having at least one provider increased; for a one unit increase in affluence, there was a predicted $525 \%$ increase (OR 5.26, 95\% CI 3.57 to 7.75) in the odds of being in the highest access ASD/ $\mathrm{BCBA}$ ratio category. However, the predicted increase in odds was less for counties in the lowest access ASD/ BCBA ratio categories. Similarly, although the relationships were much smaller, an increase in the percent of residents who were non-Hispanic white was related to an increase in the odds of a county being in the 21-30 ASD/BCBA (OR 1.02, 95\% CI 1.00 to 1.03 ) or $>30 \mathrm{ASD} /$ BCBA (OR 1.01, 95\% CI 1.00 to 1.03 ) ratio categories. The odds of a county having any BCBAs for urban nonMSAs and rural counties was smaller than the odds of counties within MSAs. For example, the odds of a county having $>30$ children with ASD/BCBA was 0.31 times and 0.01 times the odds of counties within MSAs for urban non-MSA and rural counties, respectively. Compared 
Table 1 Descriptive Statistics for County-level Geographic Access to Board Certified Behavior Analysts (BCBAs) among Children with Autism Spectrum Disorder (ASD) and County and State Factors $(\mathrm{N}=3040)$

\begin{tabular}{|c|c|c|c|c|}
\hline Variable & $\%(\mathrm{M})$ & SD & Sk & $\mathrm{Ku}$ \\
\hline \multicolumn{5}{|l|}{ Geographic access $^{a}$} \\
\hline No BCBAs ${ }^{b}$ & 52.99 & & & \\
\hline$>30 \mathrm{ASD} / \mathrm{BCBA}$ & 9.61 & & & \\
\hline 21-30 ASD/BCBA & 6.32 & & & \\
\hline 11-20 ASD/BCBA & 13.55 & & & \\
\hline$>0$ to $10 \mathrm{ASD} / \mathrm{BCBA}$ & 17.53 & & & \\
\hline \multicolumn{5}{|l|}{ County variables } \\
\hline Poverty $^{\mathrm{c}}$ & (0) & 0.9 & 0.74 & 1.30 \\
\hline Affluence $^{c}$ & $(0)$ & 0.9 & 1.36 & 3.19 \\
\hline$\%$ non-Hispanic White & 76.38 & 20.04 & -1.20 & 0.84 \\
\hline No Healthcare Insurance & 6.87 & 5.24 & 2.53 & 11.53 \\
\hline Counties within MSAs & 37.66 & & & \\
\hline Urban non-MSAs & 41.88 & & & \\
\hline Rural & 20.46 & & & \\
\hline \multicolumn{5}{|l|}{ State variables } \\
\hline Years since insurance mandate & 7.45 & 3.91 & 0.15 & -0.05 \\
\hline No age cap & 83.39 & & & \\
\hline
\end{tabular}

MSAs metropolitan statistical areas

a Iowa's 99 counties did not report CRDC data, and three counties did not have data on the number of children with ASD enrolled in schools. These counties were not included in calculations

${ }^{\mathrm{b}}$ Board Certified Behavior Analysts

${ }^{c}$ Poverty and affluence are $\mathrm{z}$-scores, so the mean will always be zero. The following values are the minimum and maximum values of poverty and affluence, respectively: $-2.14933,4.82503$ and -1.82045 , 5.47293

to counties with no BCBAs, an increase in the percent of county residents with no healthcare insurance coverage was related to a decrease in the odds of being in the 21-30 ASD/BCBA ratio category (OR 0.94, 95\% CI 0.91 to 0.97 ). In other words, the odds of a county having no BCBAs were higher for counties with higher rates of no healthcare insurance coverage. Other county and state insurance policy variables were not significantly related to $\mathrm{ASD} / \mathrm{BCBA}$ ratios.

Based on the finding that both poverty and affluence were positively associated with geographic access, we explored whether urbanicity moderated the relationship between access and these two variables. We ran one interaction model for poverty and one interaction model for affluence. The interaction effects with poverty were minor whereas the interaction effects with affluence were pronounced. As depicted in Fig. 1, as poverty rates increased, the difference between counties within MSAs and rural counties increased in the probability of having no BCBAs. However, the magnitude of the interaction was small. As shown in Fig. 2, as affluence rates increased, the difference between counties
Table 2 Parameter Estimates for Generalized Logit Models of Geographic Access to Board Certified Behavior Analysts (BCBAs) among Children with Autism Spectrum Disorder (ASD) by County and State Factors $(\mathrm{N}=3040)$

\begin{tabular}{|c|c|c|c|}
\hline Variable & $b(\mathrm{SE})$ & OR $(95 \% \mathrm{CI})$ & $p$ \\
\hline \multicolumn{4}{|l|}{ Fixed effects (SE) } \\
\hline \multicolumn{4}{|l|}{ Intercept } \\
\hline$>30 \mathrm{ASD} / \mathrm{BCBA}$ & $-0.65(0.32)$ & & 0.045 \\
\hline 21-30 ASD/BCBA & $-1.06(0.30)$ & & 0.000 \\
\hline 11-20 ASD/BCBA & $-0.72(0.26)$ & & 0.006 \\
\hline$>0$ to $10 \mathrm{ASD} / \mathrm{BCBA}$ & $-0.81(0.30)$ & & 0.010 \\
\hline \multicolumn{4}{|l|}{ Poverty } \\
\hline$>30 \mathrm{ASD} / \mathrm{BCBA}$ & $0.26(0.16)$ & $1.30(0.94-1.77)$ & 0.111 \\
\hline 21-30 ASD/BCBA & $0.26(0.17)$ & $1.30(0.93-1.81)$ & 0.123 \\
\hline 11-20 ASD/BCBA* & $0.49(0.16)$ & $1.63(1.19-2.23)$ & 0.003 \\
\hline$>0$ to $10 \mathrm{ASD} / \mathrm{BCBA}^{*}$ & $0.56(0.16)$ & $1.76(1.26-2.44)$ & 0.001 \\
\hline \multicolumn{4}{|l|}{ Affluence } \\
\hline$>30$ ASD/BCBA* & $0.81(0.17)$ & $2.26(1.59-3.21)$ & $<0.001$ \\
\hline 21-30 ASD/BCBA* & $1.06(0.18)$ & $2.88(2.02-4.10)$ & $<0.001$ \\
\hline 11-20 ASD/BCBA* & $1.28(0.18)$ & $3.61(2.53-5.14)$ & $<0.001$ \\
\hline$>0$ to $10 \mathrm{ASD} / \mathrm{BCBA}^{*}$ & $1.66(0.19)$ & $5.26(3.57-7.75)$ & $<0.001$ \\
\hline \multicolumn{4}{|l|}{$\%$ Non-Hispanic White } \\
\hline$>30 \mathrm{ASD} / \mathrm{BCBA}^{*}$ & $0.01(0.01)$ & $1.01(1.00-1.03)$ & 0.048 \\
\hline 21-30 ASD/BCBA* & $0.02(0.01)$ & $1.02(1.00-1.03)$ & 0.020 \\
\hline 11-20 ASD/BCBA & $0.01(0.01)$ & $1.01(1.00-1.03)$ & 0.073 \\
\hline$>0$ to $10 \mathrm{ASD} / \mathrm{BCBA}$ & $0.01(0.01)$ & $1.01(1.00-1.02)$ & 0.464 \\
\hline \multicolumn{4}{|l|}{$\begin{array}{l}\% \text { No healthcare insur- } \\
\text { ance }\end{array}$} \\
\hline$>30 \mathrm{ASD} / \mathrm{BCBA}$ & $-0.03(0.02)$ & $0.97(0.93-1.02)$ & 0.236 \\
\hline 21-30 ASD/BCBA* & $-0.06(0.01)$ & $0.94(0.91-0.97)$ & 0.000 \\
\hline 11-20 ASD/BCBA & $-0.03(0.06)$ & $0.97(0.94-1.00)$ & 0.075 \\
\hline$>0$ to $10 \mathrm{ASD} / \mathrm{BCBA}$ & $-0.01(0.01)$ & $0.99(0.97-1.02)$ & 0.648 \\
\hline \multicolumn{4}{|l|}{ Urban non-MSA } \\
\hline$>30 \mathrm{ASD} / \mathrm{BCBA}^{*}$ & $-1.19(0.22)$ & $0.31(0.20-0.48)$ & $<0.001$ \\
\hline 21-30 ASD/BCBA* & $-1.07(0.20)$ & $0.34(0.23-0.51)$ & $<0.001$ \\
\hline 11-20 ASD/BCBA* & $-1.03(0.16)$ & $0.36(0.26-0.49)$ & $<0.001$ \\
\hline$>0$ to $10 \mathrm{ASD} / \mathrm{BCBA}^{*}$ & $-0.76(0.16)$ & $0.47(0.33-0.65)$ & $<0.001$ \\
\hline \multicolumn{4}{|l|}{ Rural } \\
\hline$>30 \mathrm{ASD} / \mathrm{BCBA}^{*}$ & $-4.54(0.79)$ & $0.01(0.00-0.05)$ & $<0.001$ \\
\hline 21-30 ASD/BCBA* & $-4.08(0.64)$ & $0.02(0.01-0.06)$ & $<0.001$ \\
\hline 11-20 ASD/BCBA* & $-3.06(0.47)$ & $0.05(0.02-0.12)$ & $<0.001$ \\
\hline$>0$ to $10 \mathrm{ASD} / \mathrm{BCBA}^{*}$ & $-1.87(0.30)$ & $0.15(0.08-0.28)$ & $<0.001$ \\
\hline
\end{tabular}

Reference group for the outcome, geographic access, is no BCBAs in a county. Counties within metropolitan statistical areas (MSAs) is the reference group for urban non-MSAs and rural. Model also included the variables Years Since Insurance Mandate and No Age Cap, which were not statistically significant

$S E$ standard error, $O R$ odds ratio, $C I$ confidence interval

within MSAs and rural counties also increased in the probability of having no BCBAs. The magnitude of the interaction was large. 


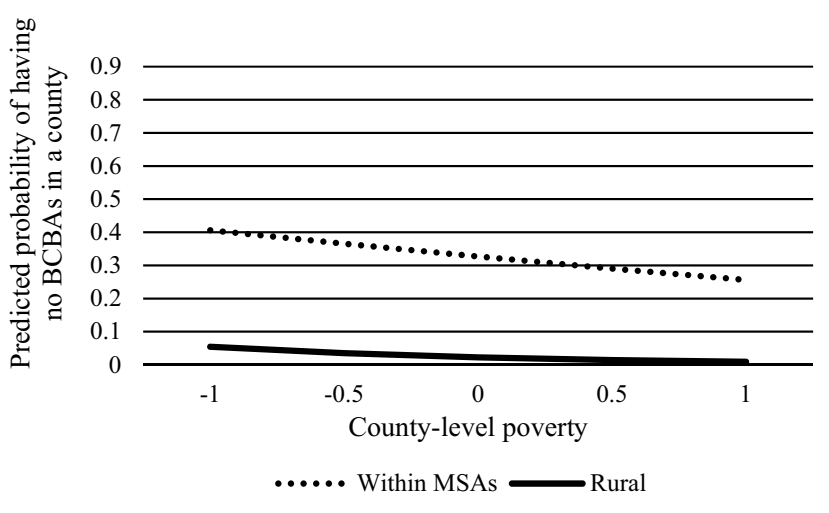

Fig. 1 The moderating effect of urbanicity on the relationship between county poverty and the probability of a county having no Board Certified Behavior Analysts. Note: Poverty is a composite variable calculated as a mean $\mathrm{z}$-score computed from variables commonly used to measure poverty: percent of children below the federal poverty level, percent of households who received supplemental security income, cash public assistance income or supplemental nutrition assistance program benefits in the past 12 months, and percent of residents who are unemployed. As poverty rates increased, the difference between counties within MSAs and rural counties increased in the probability of having no BCBAs

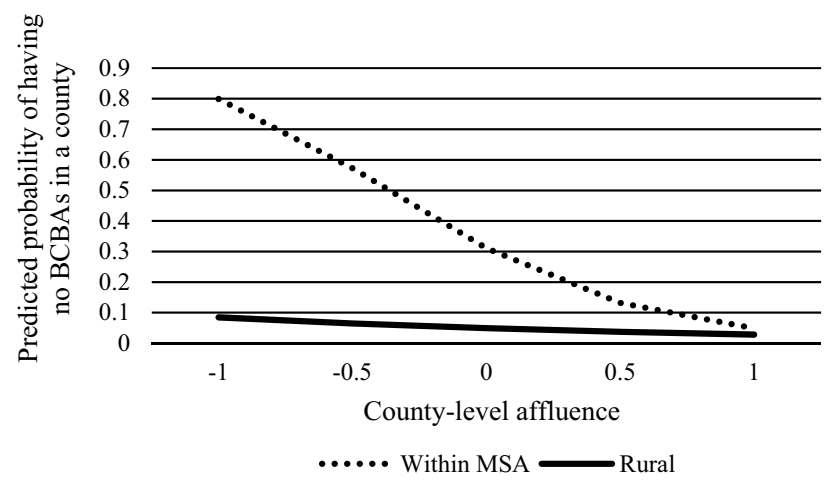

Fig. 2 The moderating effect of urbanicity on the relationship between county affluence and the probability of a county having no Board Certified Behavior Analysts. Note. Affluence is a z-score computed from variables often used to measure affluence: median household income, percent of residents with professional/managerial employment, and percent of residents 25 years or older with a Bachelor's degree or higher. As affluence rates increased, the difference between counties within MSAs and rural counties also increased in the probability of having no BCBAs

\section{Discussion}

In this study we investigated the association between geographic access to BCBAs and county and state factors. As expected, results indicate that in general, counties characterized by greater social and economic advantage are associated with higher geographic access to BCBAs. Although the relationship was not as strong as affluence, higher poverty rates were also associated with higher access. Patterns for poverty and affluence interactions were similar across different levels of the outcome. Highly affluent and urban counties had the highest access to BCBAs. Thus, urbanicity was a significant moderating factor, and affluence had a large linear impact with odds ratio estimates ranging from 2.26 to 5.26. Based on primary and supplemental analyses, higher geographic access in high poverty areas may be the result of concentrated poverty in cities, and findings suggest that targeting non-urban and less affluent counties for provider recruitment and maintenance could most improve access to BCBAs for children with ASD.

Compared to counties with no BCBAs, counties with higher rates of non-Hispanic white residents had higher geographic access to BCBAs. One potential explanation for this statistically significant finding could be the racialethnic composition of the profession. Specifically, it is possible that more providers identify as non-Hispanic white and therefore are more likely to be present in areas with higher rates of non-Hispanic white residents. Although the BACB reports new data on racial-ethnic composition that closely aligns with that of the U.S. population, to date, only $53 \%$ of BCBAs have provided this data (Behavior Analyst Certification Board 2020a, 2020a). Actual composition could be less representative than reported. Regardless, odds ratios and confidence intervals suggest a lack of clinical significance. Although evidence that high-minority counties experience low access may justify efforts to improve provider supply in those areas (Cook et al. 2013), further research is necessary before meaningful conclusions can be drawn.

Evidence that rural counties lack access may underscore the need to consider new and traditional strategies to improve access in these areas. Strategies employed to address inequity in the distribution of other providers (e.g., physicians) may have utility. Common strategies include scholarships and loan repayment programs that incentivize providers to practice in underserved locations (National Conference of State Legislatures 2017). However, research on medical students indicates that recruiting rural students may be key to developing a rural workforce (MacQueen et al. 2018). Importantly, the legitimacy of online Master's programs is increasing, and if reports on graduates in other health-related disciplines are any indication, these graduates may be more likely to reside and work in small towns or rural areas and to work with individuals covered by Medicaid (George Washington University Health Workforce Institute, n.d.). Thus, bolstering online education in behavior analysis may be one pathway to better access. Relatedly, BCBAs and their representative organizations could partner with rural schools and build capacity for telehealth (Behavior Analyst Certification Board 2019c), for which early evidence is promising (Lindgren et al. 2016; Suess et al. 2016; Vismara et al. 2018). 
During COVID-19, the acceptability of and capacity for delivering distance education and telehealth services as strategies for improving access to BCBAs may be increasing. However, there are challenges that would require consideration. One of the largest obstacles to producing BCBAs in locations with low access is providing an avenue for providers to meet the fieldwork hour requirement to become eligible to take the BCBA certification exam. With limited exceptions, before a provider can take their BCBA exam they must first accumulate a certain number of fieldwork hours while being supervised by a qualified BCBA. This supervisor must meet with the provider and observe their interactions with clients a predetermined amount of time each month for fieldwork hours to count towards eligibility requirements. Whereas some of these requirements may be handled remotely (online supervision) or rely on the BACB's recent waiver of client observation requirements due to the pandemic (COVID-19 Updates from the BACB 2020), there are privacy issues related to FERPA and HIPAA that may make it difficult for providers to meet these requirements while working with distance supervisors.

A potentially important factor related to the education of BCBAs that could not be included in analyses was the presence of Association for Behavior Analysis International Verified Course Sequences (VCS) across counties. Healthcare providers interested in becoming eligible to take a BCBA certification exam must first meet predetermined coursework, fieldwork, and degree requirements. Although not required, most providers complete these coursework requirements through a training program that offers a VCS. Importantly, VCS status is not accreditation. Instead, the purpose of VCS is to expedite the application process for providers attempting to demonstrate to the BACB that they have met the coursework eligibility requirements when applying for their certification exam. In the current study, we could not account for VCSs because there were too few to analyze. To provide further context for study results, however, the distribution of these programs across the U.S. are depicted in Fig. 3. In addition to type of program an institution of higher education offers (i.e., on-campus, hybrid, distance education, and both on-campus and distance education), we show counties without programs and without BCBAs and counties without programs and with BCBAs. Importantly, although VCS programs are available across states, these programs produce applicants taking the BCBA certification exam at very uneven rates. For example, between 2014 and 2018, more than $50 \%$ of the first-time applicants for the BCBA examination came from VCSs housed within only eight programs (Behavior Analyst Certification Board 2019b). However, given the exponential increase in the number of newly certified BCBAs each month (Behavior Analyst Certification Board 2020a), the increasing number of VCSs and online behavior analysis programs, geographic accessibility of BCBAs may improve over time. Yet this is not guaranteed and considering the very uneven distribution of BCBAs detected in this study, future research is warranted.
Fig. 3 Distribution of Association for Behavior Analysis International Verified Course Sequences across the U.S

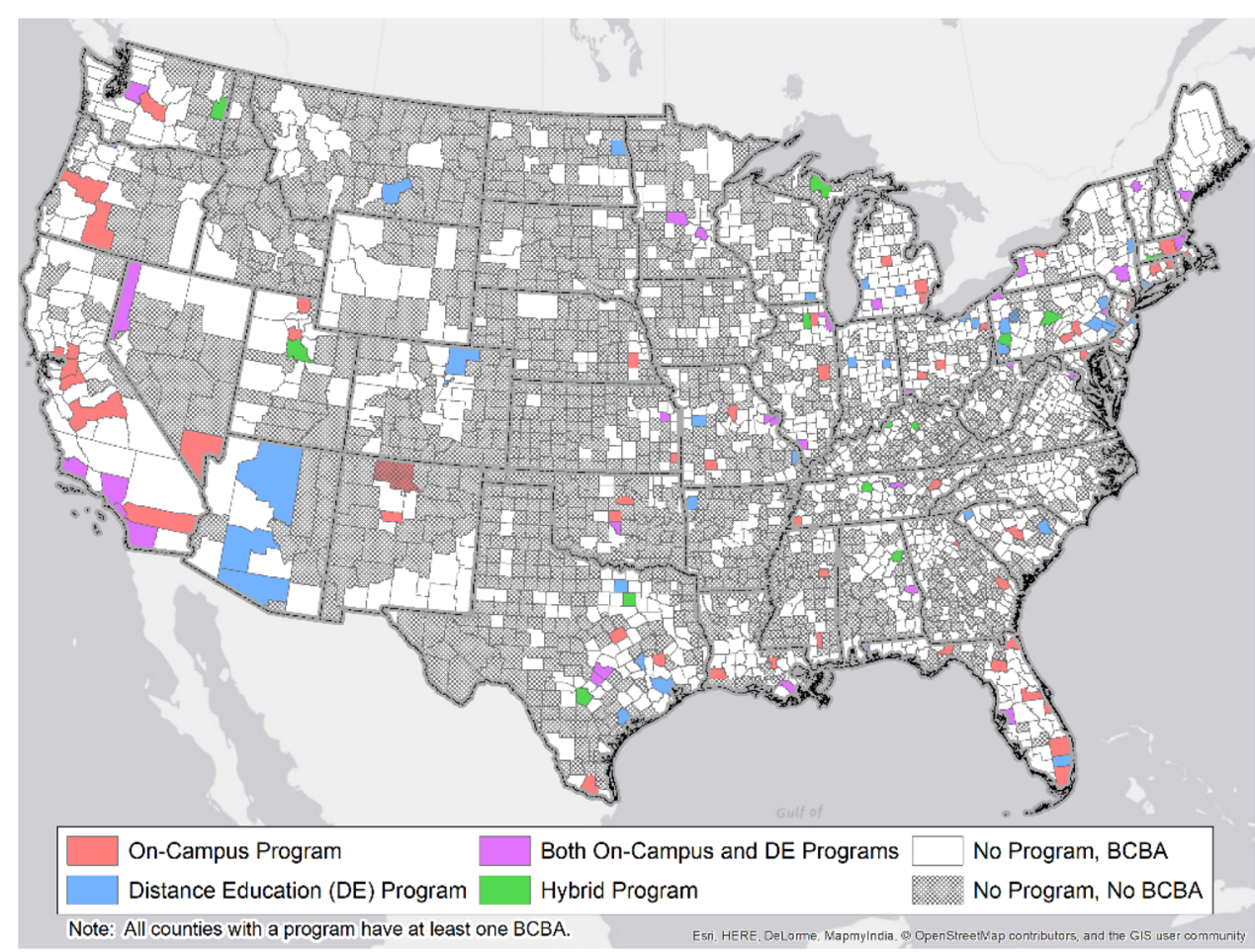


Compared to counties with no BCBAs, counties with higher rates of no healthcare insurance had less access. This finding is not surprising, as most BCBAs rely on reimbursement from health insurance for their services. If this option is not available, then BCBAs may not be able to offer services to individuals regardless of need. Despite the association between healthcare insurance at the county level and access, there was not a significant association between state insurance mandate policies and access. Specifically, the number of years since a state's insurance mandate passed and whether a county was in a state with a mandate that had no age cap did not impact access. This suggests that these policies may not influence where BCBAs practice and that other factors not included in this study may be important to examine. For example, other limitations included in a state's healthcare insurance mandate may directly impact the treatment model being used by behavioral providers. Many state insurance mandates include an annual dollar cap per patient for behavioral services. These dollar caps may not make it financially viable for BCBAs to offer medically necessary early intensive behavioral intervention services to individuals diagnosed with ASD. Instead, providers in these states may be forced to offer fewer hours of treatment or adopt a consultative treatment model to ensure reimbursement for their services. Fortunately, there is some evidence that states that originally adopted these caps are revising their mandates based on interpretations of federal laws like the Mental Health Parity and Addiction Equity Act of 2008 (Peterson and Busch 2018). Additionally, state healthcare insurance mandates only cover a subset of policies. Most significantly, these policies do not cover self-insured, or self-funded, group health plans offered by employers. An estimated $61 \%$ of covered workers fall under these plans (Claxton et al. 2018), which are regulated by federal law (e.g., ERISA) and are exempt from these state mandates. Finally, the percentage of BCBA services being funded due to these insurance mandates is still unknown. BCBAs may be relying on other funding mechanisms (e.g., TRICARE, Medicaid, Medicaid Waivers) that may reimburse at differing rates depending on the state.

\section{Limitations}

There are two primary data limitations that must be considered in the context of study findings. First, it is likely that results represent the ideal scenario for county-level geographic access to BCBAs. The CRDC does not include all children diagnosed with ASD in a school district, likely underestimating how many children with ASD need services a BCBA can provide. States only report children's primary educational disability, so it is possible that some children have a secondary disability of ASD and a different primary disability, such as ID. Importantly, states vary in requirements for a primary disability of ASD (MacFarlane \& Kanaya 2009). Additionally, children two years of age or younger and home-schooled children are excluded. Although public pre-schools are included, children with ASD for whom there is no publicly funded pre-school equipped to support their specialized needs would not be represented in the data. These children may remain at home until kindergarten. They may also receive home and/or clinic-based therapeutic services, including ABA services. Notably, children attending private schools would also not be represented. Still, the CRDC provides the best data available to estimate national prevalence of ASD, and at least $95 \%$ of students with educational disabilities are enrolled in regular public schools.

Second, not captured in the data are details about the practices of individual BCBAs. Some may not actively practice (e.g., academicians), and some may include children with other diagnoses on their caseloads. Data also were unavailable on which treatment models BCBAs use, the size and characteristics of their caseloads, and how much of their time is spent supervising staff. Furthermore, the county in which BCBAs work may differ from their county of residence, including BCBAs who provide telehealth services for children who live in rural areas.

\section{Conclusion}

This study provides preliminary evidence of sociodemographic inequities in geographic access to BCBAs in the U.S. Findings suggest that non-urban and less affluent counties experience low access and that targeted efforts to improve access in these counties could be worthwhile for children with ASD and their families. Moreover, as the number of BCBAs continues to rapidly increase (Behavior Analyst Certification Board 2020a, b), it will be prudent to monitor how geographic access changes over time.

Acknowledgements We acknowledge the Behavior Analyst Certification Board for maintaining up-to-date certificant registry data.

Funding The authors did not receive support from any organization for the submitted work. No funding was received to assist with the preparation of this manuscript. No funding was received for conducting this study. No funds, grants, or other support was received.

\section{Compliance with Ethical Standards}

Conflict of interest The authors have no relevant financial or non-financial interests to disclose. The authors have no conflicts of interest to declare that are relevant to the content of this article. All authors certify that they have no affiliations with or involvement in any organization or entity with any financial interest or non-financial interest in the subject matter or materials discussed in this manuscript. The authors 
have no financial or proprietary interests in any material discussed in this article.

\section{References}

American Psychiatric Association. (2013). Diagnostic and statistical manual of mental disorders: DSM-5. (5th edn.). American Psychiatric Association.

Andrilla, C. H. A., Patterson, D. G., Garberson, L. A., Coulthard, C., \& Larson, E. H. (2018). Geographic variation in the supply of selected behavioral health providers. American Journal of Preventive Medicine, 54(6), S199-S207. https://doi.org/10.1016/j.amepr e.2018.01.004.

Baio, J., Wiggins, L., Christensen, D. L., Maenner, M. J., Daniels, J., Warren, Z., Kurzius-Spencer, M., Zahorodny, W., Robinson, C., Rosenberg, White, T., Durkin, M. S., Imm, P., Nikolaou, L., Yeargin-Allsopp, M., Lee, L.-C., Harrington, R., Lopez, M., Fitzgerald, R. T., ... Dowling, N. F. (2018). Prevalence of Autism Spectrum Disorder among children aged 8 years-Autism and Developmental Disabilities Monitoring Network, 11 Sites, United States, 2014. MMWR. Surveillance Summaries, 67(6), 1-23. https://doi.org/https://doi.org/10.15585/mmwr.ss6706a1

Behavior Analyst Certification Board. (2014). Applied behavior analysis treatment of autism spectrum disorder: Practice guidelines for healthcare funders and managers, 2 nd edition (p. 46). https ://www.bacb.com/wp-content/uploads/2017/09/ABA_Guidelines _for_ASD.pdf.

Behavior Analyst Certification Board. (2019a). BACB Certificant Data. https://bacb.com/services/o.php?page $=101134$

Behavior Analyst Certification Board. (2019b). BCBA Examination Pass Rates for Verified Course Sequences: 2014-2018. Author. https://www.bacb.com/wp-content/uploads/BCBA-Pass-Rates -Alpha_190813.pdf

Behavior Analyst Certification Board. (2019c). Clarifications regarding applied behavior analysis treatment of autism spectrum disorder: Practice guidelines for healthcare funders and managers (2nd ed.). Author.

Behavior Analyst Certification Board. (2020a). BACB Certificant Data. https://bacb.com/services/o.php?page $=101134$

Behavior Analyst Certification Board. (2020b). US employment demand for behavior analysts: 2010-2019. Author. https://www. bacb.com/wp-content/uploads/US-Employment-Demand-forBehavior-Analysts_2020_.pdf

Brown, E. J., Polsky, D., Barbu, C. M., Seymour, J. W., \& Grande, D. (2016). Racial disparities in geographic access to primary care in Philadelphia. Health Affairs, 35(8), 1374-1381. https://doi. org/10.1377/hlthaff.2015.1612.

Carr, J. E., \& Nosik, M. R. (2017). Professional credentialing of practicing behavior analysts. Policy Insights from the Behavioral and Brain Sciences, 4(1), 3-8. https://doi.org/10.1177/2372732216 685861.

Centers for Medicare and Medicaid Services. (2014). Medicaid and CHIP FAQs: Services to address Autism. Department of Health and Human Services. http://www.medicaid.gov/federal-polic y-guidance/downloads/faq-09-24-2014.pdf

Claxton, G., Rae, M., Long, M., Damico, A., \& Whitmore, H. (2018). Health benefits in 2018: Modest growth in premiums, higher worker contributions at firms with more low-wage workers. Health Affairs, 37(11), 1892-1900. https://doi.org/10.1377/hltha ff.2018.1001.

Cook, B. L., Doksum, T., Chen, C., Carle, A., \& Alegría, M. (2013). The role of provider supply and organization in reducing racial/ ethnic disparities in mental health care in the U.S. Social Science
\& Medicine (1982), 84, 102-109. https://doi.org/https://doi. org/10.1016/j.socscimed.2013.02.006

COVID-19 Updates from the BACB. (2020). Behavior Analyst Certification Board. https://www.bacb.com/bacb-covid-19-updates/

Cummings, J., Wen, H., Ko, M., \& Druss, B. (2013). Geography and the Medicaid mental health care infrastructure: Implications for health care reform. JAMA Psychiatry, 70(10), 1084-1090.

Davis, M. A., Anthopolos, R., Tootoo, J., Titler, M., Bynum, J. P. W., \& Shipman, S. A. (2018). Supply of healthcare providers in relation to county socioeconomic and health status. Journal of General Internal Medicine, 33(4), 412-414. https://doi.org/10.1007/s1160 6-017-4287-4.

Deochand, N., \& Fuqua, R. W. (2016). BACB certification trends: State of the states (1999 to 2014). Behavior Analysis in Practice, 9(3), 243-252. https://doi.org/10.1007/s40617-016-0118-z.

George Washington University Health Workforce Institute. (n.d.). A comparison of in person and online Master's of Social Work graduates: Demograhpic and educational background, the jobs they take, and the populations they serve. Retrieved March 21, 2019, from https://cswe.org/getattachment/Centers-Initiatives/Initi atives/National-Workforce-Initiative/Online-vs-Inperson-Workf orce-Data-Brief-2018-(1).pdf.aspx

Goodchild, M. F., \& Lam, N.S.-N. (1980). Areal interpolation: A variant of the traditional spatial problem. Geoprocessing, 1, 297-331.

Goodman, D. C., Fisher, E. S., Little, G. A., Stukel, T. A., Chang, C., \& Schoendorf, K. S. (2002). The relation between the availability of neonatal intensive care and neonatal mortality. The New England Journal of Medicine, 7.

Guttmann, A., Shipman, S. A., Lam, K., Goodman, D. C., \& Stukel, T. A. (2010). Primary care physician supply and children's health care use, access, and outcomes: Findings from Canada. Pediatrics, 125(6), 1119-1126. https://doi.org/10.1542/peds.2009-2821.

Kalkbrenner, A. E., Daniels, J. L., Emch, M., Morrissey, J., Poole, C., \& Chen, J.-C. (2011). Geographic access to health services and diagnosis with an autism spectrum disorder. Annals of Epidemiology, 21(4), 304-310. https://doi.org/10.1016/j.annep idem.2010.11.010.

Kennedy-Hendricks, A., Epstein, A. J., Mandell, D. S., Candon, M. K., Marcus, S. C., Xie, M., \& Barry, C. L. (2018). Effects of state autism mandate age Caps on health service use and spending among adolescents. Journal of the American Academy of Child \& Adolescent Psychiatry, 57(2), 125-131. https://doi.org/10.1016/j. jaac.2017.10.019.

Ko, M., Cummings, J., \& Ponce, N. (2016). Changes in the supply of US rural health centers, 2000-2011: Implications for rural minority communities. The Journal of Rural Health, 32(3), 245-253.

Leventhal, T., \& Brooks-Gunn, J. (2003). Children and youth in neighborhood contexts. Current Directions in Psychological Service, 12(1), 27-31.

Lindgren, S., Wacker, D., Suess, A., Schieltz, K., Pelzel, K., Kopelman, T., et al. (2016). Telehealth and autism: Treating challenging behavior at lower cost. Pediatrics, 137(Supplement), S167-S175. https://doi.org/10.1542/peds.2015-28510.

MacFarlane, J. R., \& Kanaya, T. (2009). What does it mean to be Autistic? Inter-state variation in special education critieria for Autism services. Journal of Child and Family Studies, 18(6), 662-669.

MacQueen, I. T., Maggard-Gibbons, M., Capra, G., Raaen, L., Ulloa, J. G., Shekelle, P. G., et al. (2018). Recruiting rural healthcare providers today: A systematic review of training program success and determinants of geographic choices. Journal of General Internal Medicine, 33(2), 191-199. https://doi.org/10.1007/s1160 6-017-4210-z.

Markoff, J., \& G, S. . (1973). The linkage of data describing overlapping geographical units. Historical Methods Newsletter, 7, 34-46.

McBain, R. K., Kareddy, V., Cantor, J. H., Stein, B. D., \& Yu, H. (2019). Systematic review: United States workforce for 
Autism-related child healthcare services. Journal of the American Academy of Child \& Adolescent Psychiatry. https://doi. org/10.1016/j.jaac.2019.04.027.

Mello, M. P., Urbano, R. C., Goldman, S. E., \& Hodapp, R. M. (2016). Services for children with autism spectrum disorder: Comparing rural and non-rural communities. Education and Training in Autism and Developmental Disabilities, 51(4), 355.

National Autism Center. (2015). Findings and conclusions: National standards project, phase 2. Randolph, MA: Author.

National Conference of State Legislatures. (2017, August 30). Health Professional Shortage Areas, 2017 postcard. http://www.ncsl.org/ research/health/health-professional-shortage-areas-2017-postcard. aspx

National Conference of State Legislatures. (2019). Autism and insurance coverage state laws. http://www.ncsl.org/research/health/ autism-and-insurance-coverage-state-laws.aspx

Peterson, E., \& Busch, S. (2018). Achieving mental health and substance use disorder treatment parity: A quarter century of policy making and research. Annual Review of Public Health, 39, 421435. https://doi.org/10.1146/annurev-publhealth-040617-013603.

Peters-Scheffer, N., Didden, R., Korzilius, H., \& Sturmey, P. (2011). A meta-analytic study on the effectiveness of comprehensive ABA-based early intervention programs for children with autism spectrum disorders. Research in Autism Spectrum Disorders, 5(1), 60-69. https://doi.org/10.1016/j.rasd.2010.03.011.

Rosenblatt, R., Chen, F., Lishner, D., \& Doescher, M. (2010). The future of family medicine and implications for rural primary care physician supply (Final Report \#125). WWAMI Rural Health Research Center, University of Washington School of Medicine Department of Family Medicine. https://depts.washington.edu/ uwrhrc/uploads/RHRC_FR125_Rosenblatt.pdf

Roth, M., \& DiGennaro-Reed, F. (2014). A meta-analysis of behavioral interventions for adolescents and adults with Autism Spectrum Disorders. Journal of Behavioral Education, 23, 258-286.

Shipman, S. A., Lan, J., Chang, C., \& -h., \& Goodman, D. C. . (2011). Geographic maldistribution of primary care for children. Pediatrics, 127(1), 19-27. https://doi.org/10.1542/peds.2010-0150.

State regulated health benefit plans. (2020). Autism Speaks. https:// www.autismspeaks.org/state-regulated-health-benefit-plans

Suess, A., Wacker, D., Schwartz, J., Lustig, N., \& Detrick, J. (2016). Preliminary evidence on the use of telehealth in an outpatient behavior clinic. Journal of Applied Behavior Analysis, 49(3), 686-692.

United States Department of Agriculture. (2019, October 25). USDA Economic Research Service-Rural-Urban Continuum Codes. Rural-Urban Continuum Codes [Data Set]. https://www.ers.usda. gov/data-products/rural-urban-continuum-codes//

United States Department of Education. (2018). 2017-18 Civil Rights Data Collection: List of CRDC data elements for school year 2017-18. https://www2.ed.gov/about/offices/list/ocr/docs/201718-crdc-data-elements.pdf

US Department of Education, Institute for Education Sciences. (2016). Functional behavioral assessment-based interventions. What Works Clearinghouse. https://ies.ed.gov/ncee/wwc/Interventi on/1241

Virués-Ortega, J. (2010). Applied behavior analytic intervention for autism in early childhood: Meta-analysis, meta-regression and dose-response meta-analysis of multiple outcomes. Clinical Psychology Review, 30(4), 387-399. https://doi.org/10.1016/j. cpr.2010.01.008.

Vismara, L. A., McCormick, C., Wagner, A., Monlux, K., Nadhan, A., \& Young, G. (2018). Telehealth parent training in the Early Start Denver Model: Results from a randomized controlled study. Focus on Autism and Other Developmental Disabilities, 33(2), 67-79.

Wong, C., Odom, S. L., Hume, K., Cox, A. W., Fettig, A., Kucharczyk, S., Brock, M. E., Plavnick, J., Fleury, V., \& Schultz, T. (2014). Evidence-based practices for children, youth, and young adults with Autism Spectrum Disorder. Chapel Hill: The University of North Carolina, Frank Porter Graham Child Development Institute, Autism Evidence-Based Practice Review Group.

Yingling, M. E., Bell, B. A., \& Hock, R. M. (2019). Treatment utilization trajectories among children with autism spectrum disorder: Differences by race-ethnicity and neighborhood. Journal of Autism and Developmental Disorders, 49(5), 2173-2183. https:// doi.org/10.1007/s10803-019-03896-3.

Publisher's Note Springer Nature remains neutral with regard to jurisdictional claims in published maps and institutional affiliations. 